\title{
FUNCTIONAL ANALYSIS AND NONLINEAR DIFFERENTIAL EQUATIONS ${ }^{1}$
}

\author{
BY L. CESARI AND R. KANNAN \\ Communicated by Frederick Gehring, June 12, 1973
}

1. The aim of this paper is to study the nonlinear differential equation (1)

$$
E x=N x
$$

where $N$ is a nonlinear operator in a real Hilbert space $S$, and $E$ is a linear differential operator in $S$ with preassigned linear homogeneous boundary conditions. The idea is to reduce the problem to a finite dimensional setting and this technique has been used by several authors. We use here a method due to Cesari [4]. This method has been extensively developed in the existence analysis of differential equations by Cesari, Hale, Locker, Mawhin and others. For a detailed bibliography one is referred to Cesari [5].

In this paper, by applying results from the theory of monotone operators, we show that, under suitable monotonicity hypotheses on $N$, the equation $E x=N x$ can be solved. In the present short presentation we restrict ourselves to the simplest hypotheses on $E, N$ and $S$, even though the results obtained here hold under more general conditions.

2. Let $S$ be the direct sum of the subspaces $S_{0}$ and $S_{1}$ and let $P: S \rightarrow S_{0}$ be a projection operator with null space $S_{1}$, and $H: S_{1} \rightarrow S_{1}$ a linear operator such that $\left(\mathrm{h}_{1}\right) H(I-P) E x=(I-P) x, x$ belonging to the domain of $E$. If $y$ is a solution of (1), then $E y=N y$ implies $H(I-P) E y=$ $H(I-P) N y$. Hence, $(I-P) y=H(I-P) N y$; and finally

$$
y=P y+H(I-P) N y .
$$

Thus, any solution of (1) is a solution of (2). If we also have that $\left(\mathrm{h}_{2}\right) E P x=P E x$ and $\left(\mathrm{h}_{3}\right) E H(I-P) N x=(I-P) N x$, then from (2) we derive

$$
E y=E P y+E H(I-P) N y=P E y+(I-P) N y .
$$

Hence, $E y-N y=P(E y-N y)$. Thus, any solution $y$ of (2) is a solution of (1) if and only if $y$ satisfies

$$
P(E y-N y)=0 .
$$

\footnotetext{
AMS (MOS) subject classifications (1970). Primary $47 \mathrm{H} 15$.

${ }^{1}$ Part of the work was done when the second author was visiting the University of Michigan, in the frame of US-AFOSR Project 71-2122.
} 
Thus, under hypotheses $\left(\mathrm{h}_{1}\right),\left(\mathrm{h}_{2}\right)$, and $\left(\mathrm{h}_{3}\right)$, we have

THEOREM. An element $y$ in $S$ is a solution of (1) if and only if $y$ is a solution of equations (2) and (3).

Equations (2) and (3) are called the auxiliary and bifurcation equations respectively. Note that, if $S$ is a separable Hilbert space with norm $\|x\|^{2}=\langle x \cdot x\rangle$ and $\left(\phi_{1}, \phi_{2}, \ldots\right)$ as an orthonormal basis, and we consider $S_{0}$ as spanned by $\left\{\phi_{1}, \phi_{2}, \ldots, \phi_{m}\right\}$, then (3) reduces to the finite system of equations $(E y-N y) \cdot \phi_{i}=0, i=1,2, \ldots, m$.

3. Let us assume that the associated linear problem $E x+\lambda x=0$ (with preassigned linear homogeneous boundary conditions) has a countable system of eigenvalues $\lambda_{i}$ and eigenfunctions $\phi_{i}$ such that $\lambda_{i} \leqq \lambda_{i+1}, \lambda_{i} \rightarrow+\infty$ as $i \rightarrow \infty$ and $\left\{\phi_{i}\right\}$ is a complete orthonormal system in the Hilbert space $S=L_{2}(A)$ of all square integrable functions $x(\alpha), \alpha \in A$.

Any element $x \in S$ can be written as $\sum c_{i} \phi_{i}$.

Let $P x=\sum_{1}^{m} c_{i} \phi_{i}$ and $E x=-\sum_{1}^{\infty} c_{i} \lambda_{i} \phi_{i}$. Thus,

$$
(I-P) x=\sum_{m+1}^{\infty} c_{i} \phi_{i}, \quad(I-P) x \in S_{1},
$$

and for $m$ such that $\lambda_{m+1}>0$, let $H: S_{1} \rightarrow S_{1}$ be defined by $H(I-P) x=$ $-\sum_{m+1}^{\infty} c_{i} \lambda_{i}^{-1} \phi_{i}$.

It can be easily seen that $H(I-P) E x=(I-P) x, E P x=P E x$, $E H(I-P) x=(I-P) x$.

For $x=\sum c_{i} \phi_{i}$, we have

$$
\begin{aligned}
\langle-H(I-P) x, x\rangle & =\sum_{m+1}^{\infty} c_{i}^{2} \lambda_{i}^{-1} \geqq \lambda_{m+1} \sum_{m+1}^{\infty} c_{i}^{2} \lambda_{i}^{-2} \\
& =\lambda_{m+1}\|-H(I-P) x\|^{2} .
\end{aligned}
$$

Hence, the operator $-H(I-P)$ is a linear, monotone operator. Since it is bounded, it is maximal monotone.

We now use the Theorem above to solve (1). To this end, we have to solve (2) and (3) respectively. Let us first consider the auxiliary equation (2), i.e., $y=P y+H(I-P) N y$. Let $x^{*}$ be any element of $S_{0}$ and consider the equation

$$
y-H(I-P) N y=x^{*} .
$$

This equation is of the type $u+L N u=x^{*}$, where $L$ is a (linear) maximal monotone operator; it has been studied by Browder [2], Brezis [1], Kolodner [7] and several others, where $N$ is assumed to satisfy suitable monotonicity hypotheses. 
In view of the fact that $\langle-H(I-P) x, x\rangle \geqq \lambda_{m+1}\|-H(I-P) x\|^{2}$ and applying the result of Hess [6], we conclude that (4) has always a unique solution $y^{*}$ for each $x^{*} \in S_{0}$, provided $N$ is also hemicontinuous.

We now proceed to consider the bifurcation equation (3). Thus we have to solve the equation $P N y^{*}=P E y^{*}$, where $y^{*}$ is the solution of (4) corresponding to $x^{*} \in S_{0}$. But $P E y^{*}=E P y^{*}=E x^{*}$ and thus equation (3) reduces to

$$
P N[I-H(I-P) N]^{-1} x^{*}-E x^{*}=0 .
$$

Let $M=N[I-H(I-P) N]^{-1}$. And let $u=M a, v=M b$, where $a, b \in S_{0}$. Then, $u=N p, v=N q$, where $p=(I-H(I-P) N)^{-1} a$ and $q=(I-H(I-P) N)^{-1} b$. Thus

$$
\begin{aligned}
\langle u-v, a-b\rangle= & \langle N p-N q, a-b\rangle \\
= & \langle N p-N q, p-H(I-P) N p-q+H(I-P) N q\rangle \\
= & \langle N p-N q, p-q\rangle \\
& +\langle N p-N q,-H(I-P) N p+H(I-P) N q\rangle .
\end{aligned}
$$

The first term on the right hand is $\geqq 0$ because $N$ is monotone and the second is so because $-H(I-P)$ is monotone. Hence,

$$
P M=P N[I-H(I-P) N]^{-1}
$$

treated as an operator from $S_{0}$ to $S_{0}$ is monotone, for if $a, b \in S_{0}$, then $\langle P M a-P M b, a-b\rangle=\langle M a-M b, a-b\rangle$. Further, if $a \in S_{0}$, then the equation

$$
a=\left\{I+P N[I-H(I-P) N]^{-1}\right\} x
$$

reduces to $a=x+P N p$, where $p=[I-H(I-P) N]^{-1} x$, or

$$
p-H(I-P) N p+P N p=a .
$$

By arguing as before it can be shown that this equation is solvable for $p$, and thus it follows from (6) that we can find $x \in S_{0}$ such that (5) is solvable. Hence, $P N[I-H(I-P) N]^{-1}$ is maximal monotone over $S_{0}$, a finite dimensional space.

Thus we are reduced to an equation in the finite dimensional space $S_{0}$ of the form $M x^{*}-E x^{*}=0$ where $M$ is maximal monotone. If $\left\langle E x^{*}, x^{*}\right\rangle \leqq 0$, as is the case when all the $\lambda_{i}$ 's are $\geqq 0$, then the above equation is solvable. If, however, $E$ has a finite number of negative eigenvalues, then one can proceed in several ways. Thus if $\lambda_{1} \leqq \lambda_{2} \leqq$ $\cdots \leqq \lambda_{m} \leqq 0$, then one can apply Browder [3, p. 21] and conclude: If $[I-H(I-P) N]^{-1}$ is continuous and

$$
\left\langle N x_{1}-N x_{2}, x_{1}-x_{2}\right\rangle \geqq c\left\|x_{1}-x_{2}\right\|^{2}, \quad c>-\lambda_{1},
$$

then the bifurcation equation is solvable. 


\section{REFERENCES}

1. H. Brezis, Équations et inéquations non-linéaires dans les espaces vectoriels en dualité, Ann. Inst. Fourier (Grenoble) 18 (1968), fasc. 1, 115-175. MR 42 \#5113.

2. F. E. Browder, Nonlinear functional analysis and nonlinear integral equations of Hammerstein and Urysohn type, Contributions to Nonlinear Functional Analysis, Math. Research Center, Madison, Wisconsin, 1971.

3. — Problèmes non-linéaires, Séminaire de Mathématiques Supérieures, no. 15 (Été, 1965), Les Presses de l'Université de Montréal, Montréal, Que., 1966. MR 40 \# 3380.

4. L. Cesari, Functional analysis and Galerkin's method, Michigan Math. J. 11 (1964), 385-414. MR 30 \#4047.

5. - Nonlinear analysis, Bressanone, CIME lecture notes, 1972.

6. P. Hess, On nonlinear equations of Hammerstein type in Banach spaces, Proc. Amer. Math Soc. 30 (1971), 308-312. MR 43 \#7980.

7. I. I. Kolodner, Equations of Hammerstein type in Hilbert space, J. Math. Mech. 13 (1964), 701-750. MR 30 \#1415.

Department of Mathematics, University of Michigan, Ann Arbor, Michigan 48104

Department of Mathematics, University of Missouri, St. Louis, Missouri 63121 